\title{
Auger recombination rate in quantum well lasers
}

\author{
P K BASU \\ Institute of Radio Physics and Electronics, 92 Acharya Prafulla Chandra Road, \\ Calcutta 700009, India \\ MS received 27 February 1984; revised 2 March 1985
}

\begin{abstract}
Auger recombination is the dominant non-radiative process in InGaAsP quantum well lasers and is responsible for the poor temperature dependence of the threshold current density. In all recent calculations of the Auger rate the electron-electron interaction potential is taken to be either of the bulk form or an approximate form derived from it. In the present work, the rate is calculated by taking an appropriate potential valid for quasi two-dimensional electrons and the expected changes are pointed out. The calculated Auger life-time is in agreement with the values reported in the literature.
\end{abstract}

Keywords. Auger recombination; quantum well lasers; semiconductors; InGaAsP.

\section{Introduction}

In conventional double heterojunction (DH) lasers, (Kressel and Butler 1977), a thin layer of a lower bandgap semiconductor is sandwiched between two layers of a higher bandgap semi-conductor. A typical example is a GaAs laser, where two layers of AlGaAs are grown on both sides of a GaAs layer. A quantum well $(\mathrm{Qw})$ laser is a special version of a DH laser, where the thickness of the middle layer is very small, of the order of $100 \mathrm{~A}$ or less. The potential profile defined by the edges of either conduction or valence bands is shown in figure la and looks like a rectangular well. The behaviour of a confined particle in such a quantum well is discussed in almost all textbooks on quantum mechanics. In Qw structure, the motion of electrons and holes in the narrow well is quantised in the direction of the growth of the layer (denoted as $z$-direction hereafter) and the carriers behave as a two-dimensional (2D) gas. The 2D carriers move in different subbands (figure $1 \mathrm{~b}$ ) and the density of states become steplike (Ando et al 1982).

It has been established (Holonyak et al 1980) that Qw lasers offer several advantages over conventional lasers. The subband energies being a function of well thickness, emission frequencies greater than the bandgap frequency can be obtained and can also be changed by changing the thickness. The line width is sharper. However, the most promising features of $\mathrm{Qw}$ lasers are (i) the threshold current density is quite low (Tsang 1981, 1982), and (ii) the temperature dependence of threshold current density is weak (Chin et al 1980). The characteristic temperature $T_{0}$ has been found to be as high as $400 \mathrm{~K}$ or more.

InGaAs or InGaAsP Qw lasers are more attractive because of their potential use in long wavelength optical communication systems. However, the threshold current density in these lasers shows a poor temperature characteristic (Rezek et al 1980) and the value of $T_{0}$ is accordingly low. The band to band Auger effect is the most important 

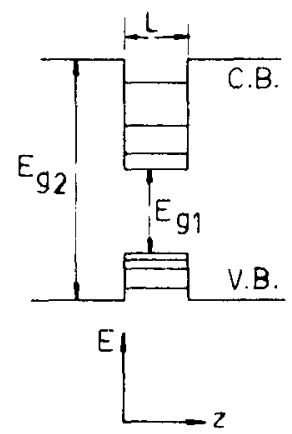

(a)
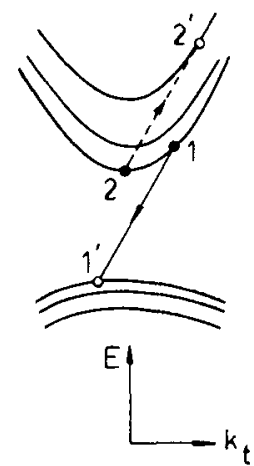

$\mathrm{CCCH}$

(b)

Figure 1. (a) Energy band diagram in a quantum well structure. $E_{q 2}\left(E_{q 1}\right)$ refers to the band gap energy of the higher (lower) band gap semiconductor. Different subbands in the quantum wells are shown. (b) A possible $\mathrm{CCCH}$ transition occurring amongst different subbands in the conduction and heavy hole bands.

process responsible for the low value of $T_{0}$. Several authors (Chiu and Yariv 1982; Dutta 1983; Sugimura 1983a, b; Smith et al 1983) have presented the calculation of Auger rates in Qw lasers. All these workers use the coulomb potential, either screened or unscreened, occurring between two electrons in bulk, to calculate the matrix element for transition. A few authors also impose restrictions on the value of $q_{z}$, the $z$-component of wave vector transfer and obtain selection rules for transitions into various subbands. It has been proved however (Ando et al 1982) that the electron-electron $(e-e)$ interaction potential in $2 D$ systems is altogether different from the screened coulomb form. The purpose of the present paper is to examine how the Auger rate calculated by previous workers is modified when an appropriate potential is introduced and also to examine the validity of the selection rules. In $\$ 2$ an outline of the theory is given. A discussion of the results is given in $\$ 3$ and the conclusions drawn in $\$ 4$.

\section{Outline of the theory}

\subsection{Earlier theories}

The various band to band Auger processes are termed the CCCH, CHHS and CHHL processes (Dutta 1983). Here we shall consider only the first one. One such transition is shown in figure 1b, where the primed (unprimed) numbers refer to the final (initial) states. The wave function for $2 D$ carriers in the $l_{1}$ th subband of the $j$ th band is given by

$$
\psi_{j l_{1}}(\mathbf{r}, \mathbf{k})=U_{j \mathbf{k}}(\mathbf{r}) \exp i \mathbf{k}_{i} \cdot \rho \sin \left(l_{1} \pi z / L\right)
$$

Here $U$ represents the localised part of Bloch function, $\mathbf{k}_{t}$ and $\rho$ are $2 D$ vectors in the plane of the layer, $L$ is the thickness of the well which is assumed to be rectangular with infinitely large height. 
Now the matrix element for transition is given by

$$
M=\iint \psi_{1^{\prime}}^{*}\left(\mathbf{r}_{1}\right) \psi_{2^{\prime}}^{*}\left(\mathbf{r}_{2}\right) V \psi_{1}\left(\mathbf{r}_{1}\right) \psi_{2}\left(\mathbf{r}_{2}\right) \mathrm{d}^{3} r_{1} \mathrm{~d}^{3} r_{2}
$$

where the band index has been suppressed. $V$ is the coulomb interaction potential between two carriers located at $\mathbf{r}_{1}$ and $\mathbf{r}_{2}$ and expressed as (Sugimura 1983a, b)

$$
V=\left(e^{2} / 4 \pi \varepsilon_{0}\left|\mathbf{r}_{1}-\mathbf{r}_{2}\right|\right) \exp \left(-\lambda\left|\mathbf{r}_{1}-\mathbf{r}_{2}\right|\right)
$$

where $\lambda$ is the inverse screening length. Equation (3) is the usual screened coulomb potential and can be written as the following Fourier series

$$
V=\frac{e^{2}}{4 \pi \varepsilon_{0}} \int \frac{4 \pi}{q^{2}+\lambda^{2}} \exp \left[i \mathbf{q} \cdot\left(\mathbf{r}_{1}-\mathbf{r}_{2}\right)\right] \mathrm{d}^{3} q
$$

Using (1) and (4) in (2) and putting $q^{2}=q_{t}^{2}+q_{z}^{2}$ one obtains integrals like

$$
\int_{-\alpha}^{\alpha} \frac{\mathrm{d} q_{z} \exp \left[i\left(k_{l_{2}} \pm k_{l_{2}}+q_{z}\right) z\right]}{q_{t}^{2}+q_{z}^{2}+\lambda^{2}}, k_{l_{2}^{\prime}}=\left(l_{2^{\prime}}, \pi / L\right)
$$

Sugimura assumed that

$$
q_{z}=\left(k_{l_{2}} \pm k_{l_{2}}\right)=\left(l_{2}, \pm l_{2}\right)(\pi / L)
$$

to calculate the above integrals. The above restriction on the value of $q_{z}$ is known as the momentum conservation approximation (MCA) introduced by Ridley (1982) to calculate the scattering rate due to electron-polar optic phonon interaction in $\mathrm{QW}$ structures. The matrix element obtained is then

$$
\begin{aligned}
M= & \frac{e^{2}}{\varepsilon_{0}} F_{11^{\prime}}, F_{22^{\prime}} \delta\left(\mathbf{k}_{1 t}+\mathbf{k}_{2 t}-\mathbf{k}_{1^{\prime} t}-\mathbf{k}_{2^{\prime} t}\right) \\
& \times \frac{2}{L^{2}}\left[\frac{\delta_{m^{+} n^{+}}+\delta_{m^{-} n^{-}}-\delta_{m^{+}\left(-n^{-}\right)}-\delta_{\left(-m^{-}\right) n^{+}}}{\left|\mathbf{k}_{2 t}-\mathbf{k}_{2{ }^{\prime}}\right|^{2}+\left(\mathbf{n}^{-} \pi / L\right)^{2}+\lambda^{2}}-\left(n^{-} \rightarrow n^{+}\right)\right. \\
F_{11^{\prime}}= & \int U_{l_{1}}^{*} U_{l_{1}} \mathrm{~d}^{2} r_{1} ; m^{ \pm}=l_{1} \pm l_{1^{\prime}} ; n^{ \pm}=l_{2} \pm l_{2^{\prime}}
\end{aligned}
$$

The presence of Kronecker deltas in (6) gives rise to selection rules governing transitions amongst various subbands. Matrix elements have been expressed by a few other workers also. Smith et al (1983) started with unscreened coulomb potential, proceeded similarly, but did not use MCA. Chiu and Yariv (1982) used the screened coulomb form but did not clearly express how they took into account $q_{z}$. Dutta (1983) started with the screened coulomb form and converted that potential into an effective $2 D$ potential by assuming the $z$-dependence of wave function to be a $\delta$-function. The matrix element then becomes

$$
\begin{aligned}
M & =\left(e^{2} / 2 \varepsilon_{0} q_{t}\right) F_{11^{\prime}} \cdot F_{22^{\prime}} G_{11^{\prime}}, G_{22^{\prime}} \delta\left(\mathbf{k}_{1 \mathrm{t}}+\mathbf{k}_{2 t^{\prime}}-\mathbf{k}_{1^{\prime} t}-\mathbf{k}_{2^{\prime} t}\right) \\
G_{11^{\prime}} & =\frac{2}{L} \int_{0}^{L} \sin \left(\frac{l_{1} \pi z_{1}}{L}\right) \sin \left(\frac{l_{1}, \pi z_{1}}{L}\right) \mathrm{d} z_{1}=1, \text { when } l_{1}=l_{1^{\prime}}=1
\end{aligned}
$$

Dutta assumed that only the lowest subbands are occupied. The above assumption leads to $q_{z}=0$, i.e., MCA and the same selection rule as proposed by Sugimura. 


\subsection{Present theory}

In the present calculation we express the potential $V$ between two carriers located at $\left(\rho_{1}, z_{1}\right)$ and $\left(\rho_{2}, z_{2}\right)$ as

$$
V=\left(e^{2} / 4 \pi \varepsilon_{0}\right)\left[\left(\rho_{1}-\rho_{2}\right)^{2}+\left(z_{1}-z_{2}\right)^{2}\right]^{-1 / 2}
$$

$V$ is written in terms of the following $2 D$ Fourier series

$$
V=\sum_{q_{t}}\left(e^{2} / 2 \varepsilon^{*} q_{t}\right) \exp \left(-q_{t}\left|z_{1}-z_{2}\right|\right) \exp \left[i q_{t} \cdot\left(\rho_{1}-\rho_{2}\right)\right]
$$

Here $\varepsilon^{*}$ is the permittivity modified by screening due to $2 D$ electrons and is approximately given by

$$
\varepsilon^{*}=\varepsilon_{0}\left(1+\frac{s}{q_{t}}\right) ; s=\frac{e^{2} n_{2 D}}{2 \varepsilon_{0} k_{B} T},
$$

$s$ being the inverse screening length in $2 D$ and $n_{2 D}$ being the sheet carrier concentration. The matrix element now becomes

$$
\begin{aligned}
& M=\delta\left(\mathbf{k}_{1 t}+\mathbf{k}_{2 t}-\mathbf{k}_{1^{\prime} t}-\mathbf{k}_{2^{\prime} t}\right)\left(\frac{e^{2}}{2 \varepsilon_{0}}\right) \frac{1}{q_{t}+s} F_{11^{\prime}} F_{22^{\prime}}, G_{11^{\prime}} G_{22^{\prime}} \\
& G_{11^{\prime}} G_{22^{\prime}}=F\left(q_{t}\right)=\frac{4}{L^{2}} \int_{0}^{L} \int_{0}^{L} \mathrm{~d} z_{1} \mathrm{~d} z_{2} \exp \left(-q_{t}\left|z_{1}-z_{2}\right|\right) \\
& \sin \left(\frac{l_{1} \pi z_{1}}{L}\right) \sin \left(\frac{l_{2} \pi z_{2}}{L}\right) \sin \left(\frac{l_{1}, \pi z_{1}}{L}\right) \sin \left(\frac{l_{2^{\prime}} \pi z_{2}}{L}\right)
\end{aligned}
$$

Straight forward integration gives

$$
\begin{aligned}
F\left(q_{t}\right)= & \frac{4}{L^{2}}\left[\frac{q_{t}\left[\delta_{m^{-} n^{-}}-\delta_{m^{-} n^{+}}\right]}{2\left[q_{t}^{2}+\left(n^{-} \pi / L\right)^{2}\right]} \frac{1}{2-\delta_{o n^{-}}}-\frac{q_{t}\left[\delta_{m^{+} n^{-}}-\delta_{m^{+} n^{+}}\right]}{2\left[q_{t}^{2}+\left(n^{+} \pi / L\right)^{2}\right]} \frac{1}{2-\delta_{o n^{+}}}\right. \\
& +\frac{q_{t}^{2}}{2}\left(e^{-q_{t} L}-1\right)\left\{\frac{1}{q_{t}^{2}+\left(n^{-} \pi / L\right)^{2}}-\frac{1}{q_{t}^{2}+\left(n^{+} \pi / L\right)^{2}}\right\} \\
& \times\left\{\frac{1}{q_{t}^{2}+\left(m^{-} \pi / L\right)^{2}}-\frac{1}{q_{t}^{2}+\left(m^{+} \pi / L\right)^{2}}\right\} \\
\mathbf{q}_{t}= & \mathbf{k}_{2 t}-\mathbf{k}_{2^{\prime} t}
\end{aligned}
$$

The first two terms in (13) may give rise to the same selection rules as proposed by Sugimura. However, the presence of the last term violates the rules.

Under the special case $l_{1}=l_{1^{\prime}}=l_{2}=l_{2^{\prime}}=1$ the matrix element may be written from (11) and (13) as

$$
\begin{aligned}
& M=\delta\left(\mathbf{k}_{1 t}+\mathbf{k}_{2 t}-\mathbf{k}_{1 \cdot t}-\mathbf{k}_{2 \cdot t}\right)\left(\frac{e^{2}}{2 \varepsilon_{0}}\right) \frac{1}{q_{t}+s} F_{11^{\prime}} F_{22^{\prime}} \\
& {\left[\frac{3 x}{x^{2}+4 \pi^{2}}+\frac{8 \pi^{2}}{x\left(x^{2}+4 \pi^{2}\right)}+\frac{32 \pi^{4}\left(e^{-x}-1\right)}{x^{2}\left(x^{2}+4 \pi^{2}\right)^{2}}\right]} \\
& x=q_{t} L
\end{aligned}
$$


Assuming that only the lowest subbands are occupied the Auger rate is given by (Dutta 1983)

$$
\begin{gathered}
R=\frac{2 \pi}{\hbar}\left(\frac{1}{4 \pi^{2}}\right)^{4} \frac{1}{L}|M|^{2} \int P\left(1,1^{\prime}, 2,2^{\prime}\right) \delta\left(E_{i}-E_{f}\right) \\
\delta\left(\mathbf{k}_{1 t}+\mathbf{k}_{2 t}-\mathbf{k}_{1^{\prime} t}-\mathbf{k}_{2^{\prime} \mathrm{t}}\right) \mathrm{d}^{2} k_{1 \mathrm{t}} \mathrm{d}^{2} k_{2 t} \mathrm{~d}^{2} k_{1^{\prime} \mathrm{t}} \mathrm{d}^{2} k_{2^{\prime} t}
\end{gathered}
$$

The integral has been evaluated by Dutta. One notes that the rate calculated by Dutta will be modified due to the difference in the matrix elements given by (7) and (14).

\section{Results and discussions}

It has been pointed out that the selection rules proposed by Sugimura break down. To estimate the error involved in the calculation when these rules are assumed valid we take the simple case, i.e., the matrix element given by (14). Note that assuming MCA and selection rules one obtains only the first term within the square bracket in (14).

Following Dutta, let us take $q_{t} \simeq k_{2^{\prime} t}=\left(2 m^{*} E_{q} / \hbar^{2}\right)^{1 / 2}, E_{q}=1.3 \mathrm{eV} ; m^{*}=0.061 m_{0}$; $L=200 \mathrm{~A}$. This yields $x \gg 2 \pi$ and only the first term within the square bracket in (14) becomes significant. If we assume further that $q_{t} \gg s$ in (14), then the expression for $M$ becomes exactly the same as that obtained from (6) by putting $q_{t} \gg \lambda$ and all the subband indices equal to unity. The matrix element $M$ varies as $q_{t}^{-2}$ then. Exactly similar dependence has also been derived by Smith et al (1983). Although the conclusions are valid for the simplest case when only the lowest subbands are occupied, they are expected to be valid in general when $x \gg(m \pi)$. Thus for the purpose of numerical computation expressions based on MCA given by Sugimura are quite accurate, as long as screening is negligible. The same conclusion does not apply to Dutta's calculation, however. It has been pointed out that the values of Auger rate calculated by Dutta should be modified by an extra factor $\left|F\left(q_{t}\right)\right|^{2}$. Taking the above reasonable values of $q_{t}$, one finds that $\left|F\left(q_{t}\right)\right|^{2} \simeq 0.016$.

It may be of interest now to compare the Auger lifetime $\tau_{A}$ calculated from the present theory with the values given by other workers. For this purpose, we take wavelength $\lambda_{0}=1.3 \mu \mathrm{m}$, corresponding to zero dispersion in optical fibres, $L=200 \AA$ and the injected carrier density $n=1 \times 10^{18} \mathrm{~cm}^{-3}$. The values obtained from the present theory will differ from Dutta's calculation by a factor $\left|F\left(q_{t}\right)\right|^{2}$. The value of $\tau_{A}=7 \mathrm{nsec}$ is obtained for the present situation from figure 5 of Dutta's paper. However, in his calculation a factor of $2 \pi$ was omitted while arriving at (3.6) and the value $\tau_{A}=7 \mathrm{nsec}$ is therefore $2 \pi$ times too high. Taking this into consideration and also noting that $\left|F\left(q_{t}\right)\right|^{2}=0.0144$, we obtain $\tau_{A}=77 \mathrm{nsec}$ from the present theory. This value is obtained by assuming the $\mathrm{CCCH}$ process to occur between only the lowest pair of subbands.

The values of $\tau_{A}$ for the cHHS process for different values of injected carrier density and of well thicknesses have also been given by Sugimura. It has been pointed out by Dutta that the Auger lifetimes for $\mathrm{CCCH}$ and $\mathrm{CHHS}$ processes are of the same order. Our results cannot be directly compared with Sugimura's values, since the latter are obtained considering multiple subband pairs. At low values of well thickness, however, the subbands are wide apart and simultaneous energy and momentum conservations are expected to be satisfied by the lowest pair of subbands only. Therefore, a 
comparison may be possible if the values for small well thicknesses given by Sugimura are extrapolated. An approximate value of $\tau_{A}=100 \mathrm{nsec}$ for $n=1 \times 10^{18} \mathrm{~cm}^{-3}$ and $\lambda_{0}$ $=1.07 \mu \mathrm{m}$ is obtained by this procedure. This yields a value of $80 \mathrm{nsec}$ for $\tau_{A}$ at $1.3 \mu \mathrm{m}$, which is in close agreement with our results.

Recently, the Auger lifetime for InGaAsP DH laser has been estimated from experimental data (Sermage et al 1983). At $\lambda_{0}=1.3 \mu \mathrm{m}$ and for $n=1 \times 10^{18} \mathrm{~cm}^{-3}$ the deduced value for $\tau_{A}$ is 55 nsec. No such estimate for $Q$ w lasers from experimental data exists at the present moment. Smith et al (1984) however, pointed out that $\tau_{A}$ should be of the same order in both the DH and Qw lasers. Our estimate for Auger lifetimes therefore is of the same order as the experimentally deduced values.

\section{Conclusions}

We have obtained the expression for the matrix element for Auger transition in QW lasers by taking a potential appropriate for quasi $2 D$ electrons. It is found that when screening is negligible, the matrix element reduces to the one obtained by assuming screened coulomb potential for bulk electrons. The estimated Auger lifetime is in agreement with the values obtained by other workers and is of the same order as the value deduced from experiment.

\section{References}

Ando T, Fowler A B and Stern F 1982 Rev. Mod. Phys. 54437

Chin R, Holonyak N Jr., Vojak B A, Hess K, Dupuis R D and Dapkus P D 1980 Appl. Phys. Lett. 3619

Chiu L C and Yariv A 1982 IEEE J. Quantum Electron. QE-18 1406

Dutta N K 1983 J. Appl. Phys. 541236

Holonyak N Jr, Kolbas R M, Dupuis R D and Dapkus P D 1980 IEEE J. Quantum Electron. QE-16 170

Kressel H and Butler J K 1977 Semiconductor lasers and heterojunction LEDS (New York: Academic) p. 4 Ridley B K 1982 J. Phys. C15 5899

Rezek E A, Holonyak N Jr and Fuller B K 1980 J. Appl. Phys. 512402

Sermage B, Eichler H J, Heritage J P, Nelson R J and Dutta N K 1983 Appl. Phys. Lett. 42259

Smith C, Abram R A and Burt M G 1983 J. Phys. C16 L-171

Smith C, Abram R A and Burt M G 1984 Electron. Lett. 20893

Sugimura A 1983a Appl. Phys. Lett. 4217

Sugimura A 1983b IEEE J. Quantum Electron. QE-19 932

Tsang W T 1981 Appl. Phys. Lett. 39786

Tsang W T 1982 Appl. Phys. Lett. 40217 